Tohoku Math. J.

66 (2014), 455-469

\title{
A CONVERGENCE OF HUNT PROCESSES ON THE RING OF $p$-ADIC INTEGERS AND ITS APPLICATION TO RANDOM FRACTALS
}

\author{
HiROSHI KANEKO* AND HisAAKI MATSUMOTO
}

(Received February 13, 2013, revised August 30, 2013)

\begin{abstract}
The convergence of stochastic processes is one of subjects founded on importance of the numerical analysis and physical models with stability. Such practical importance inspires us with vast range of interests as to on which space the convergence can be addressed and which sort of accommodated method is required for demonstrating the convergence on the space in the focus. In this article, we establish an accommodated procedure to show the convergence of Markov processes on the ring of $p$-adic integers which emerges from a construction of random fractals. As seen in other studies on the subject, the notion of generalized Mosco-convergence will be highlighted.
\end{abstract}

Introduction. The convergence of stochastic processes is one of subjects founded on the importance of the numerical analysis and physical models with stability. Such practical importance inspires us with vast range of interests as to on which space the convergence can be addressed and which sort of accommodated method is required to demonstrate the convergence.

In a progress in the Dirichlet space theory, Mosco introduced the notion of $\Gamma$-convergence to encompass the convergence in terms of Dirichlet forms. The insight showed that the convergence as bilinear forms is not always sufficient to deduce the convergence of stochastic processes and clarified which sort of extra sufficient conditions must be imposed for the rigorous justification of the phenomenon.

In [15], Kuwae and Shioya implemented the framework by complying those sufficient conditions to the case where the state space is deformed and the Dirichlet form alters. After those progresses, various cases with convergent sequence of Dirichlet forms are investigated. For instance, on typical fractals emerged as the limit of sequence of closed sets with fundamental shapes, the convergence of stochastic processes on the closed sets was figured out due to the framework. In fact, Kumagai and Sturm demonstrated in [14] a vast framework on the convergence of Hunt processes which naturally encompasses the celebrated approximation method invented by Kigami in [12], where a sequence of Dirichlet forms are introduced on such closed sets aiming at the standard Hunt process on typical fractals embedded in the Euclidean space. Recently, in [17], Suzuki studied a convergence of Markov processes arising from tree structure as in [13] and [9].

2000 Mathematics Subject Classification. Primary 31C25; Secondary 11F85.

Key words and phrases. Dirichlet spaces, $p$-adic numbers. 
In the present article, we will propose an accommodated method to deal with convergence of Hunt processes with the same spirit as Hinz in [6] so that it covers the convergence arising from construction of random fractals on the ring of $p$-adic integers where the shapes of the closed sets are deformed randomly. Since we employ Dirichlet space theory in describing a sequence of Hunt processes on the randomly deformed closed sets, an analysis on the sequence of random Dirichlet forms will be required.

In the second section, we will point out examples where convergence of Hunt processes on $\boldsymbol{Z}_{p}$ is expected to be verified and propose a framework which covers these examples. As a main object in our investigation, we will consider Markov processes which admit the characterization by Kolmogorov's equations

$$
\left\{\begin{array}{l}
\frac{d}{d t} P_{K_{f}, K_{i}}(t)=-\tilde{a}\left(K_{f}\right) P_{K_{f}, K_{i}}(t)+\sum_{j \neq f}^{\infty} \tilde{u}\left(K_{f}, K_{j}\right) P_{K_{j}, K_{i}}(t), \\
\frac{d}{d t} P_{K_{f}, K_{i}}(t)=-\tilde{a}\left(K_{i}\right) P_{K_{f}, K_{i}}(t)+\sum_{j \neq i}^{\infty} P_{K_{f}, K_{j}}(t) \tilde{u}\left(K_{j}, K_{j}\right),
\end{array}\right.
$$

on $P_{K_{f}, K_{i}}(t)=P\left(X(t) \in K_{f} \mid X(0) \in K_{i}\right)$ with reasonably given coefficients $\tilde{u}\left(K_{f}, K_{j}\right)$ and $\tilde{a}\left(K_{f}\right)$ as originally proposed by Karwowski and Vilela-Mendes in [11], by introducing disjoint balls $\left\{K_{f}\right\}$ with identical radius satisfying $\cup_{f} K_{f}=Z_{p}$. We will discuss convergence of Hunt processes associated with a construction of a random fractal. To be more precise, in accordance with the removal of randomly taken ball with the radius in the cut-out procedure in [10], we remove the ball from the support of the measure involved in the coefficients $\tilde{a}\left(K_{f}\right)$ and $\tilde{u}\left(K_{f}, K_{j}\right)$ and renormalize the measure in the coefficients so that the Markov process associated with the equation with the coefficients involving the cut-out effect is obtained. In accordance with the cut-out effect with the framework of the generalized Mosco-convergence, we will take an equivalent procedure to the cut-out within the framework of Dirichlet space and study the sequence of Hunt processes constructed as step-by-step cut-outs are processed.

In the third section, we will prepare some lemmas to prove the convergence relying the notion of KS-convergence established by Kuwae and Shioya. In the final section, sufficient conditions for the convergence of Hunt processes including condition for the tightness will be verified with the terminology in the Dirichlet space theory.

The authors would like to thank the reviewer for his or her valuable comments.

1. Convergence of Dirichlet forms. As Kumagai and Sturm studied, a natural interest on the convergence of stochastic processes can arise from improving precision level in various approximations. Since the phenomenon in the ring $Z_{p}$ of $p$-adic integers is illustrated by a randomly moving ball with the radius determined by the precision level, the sort of convergence is expected to be observed when the radius of the ball goes to zero as the ball turns to a particle. The convergence is describable by taking sequence of Dirichlet forms $k$-th term of which admits the family of functions $\mathcal{C}_{k}$ taking constant on every ball with the radius $p^{-(k+1)}$ in $\boldsymbol{Z}_{p}$ as its domain. As a fundamental observation on the convergence in such primitive phenomenon, the sequence $\left\{\mathcal{E}_{H_{k, Z}}\right\}$ of Dirichlet forms arising from the construction of a Dirichlet space on $\boldsymbol{Z}_{p}$ in [7] is expected to be covered. In fact, each of the Dirichlet forms 
admits the family of functions $\mathcal{C}_{k}$ taking constant on every ball with the radius $p^{-(k+1)}$ in $\boldsymbol{Z}_{p}$ as its domain. From the consitency of the Dirichlet forms

$$
\mathcal{E}_{H_{k+1, Z_{p}}}(u, v)=\mathcal{E}_{H_{k, Z_{p}}}(u, v)
$$

for any pair of functions $u, v \in \mathcal{C}_{k}$ in the sense of [7], we can derive

$$
\lim _{k \rightarrow \infty} \mathcal{E}_{H_{k, Z_{p}}}(u, v)=\mathcal{E}_{K}(u, v)
$$

for any locally constant functions $u, v$ on $\boldsymbol{Z}_{p}$, where $\mathcal{E}_{K}(u, v)$ stands for the bilinear form in the article.

For a coverage to deal with this sort of convergence, we may focus on the sequence $\left\{\left(\mathcal{E}_{k}, \mathcal{C}_{k}\right)\right\}$ of pairs of symmetric bilinear forms and their domains each of which admits the representation

$$
\mathcal{E}_{k}(u, v)=\iint_{\left(F_{k} \times F_{k}\right) \cap\{x \neq y\}}(u(x)-u(y))(v(x)-v(y)) \rho_{k}(x, y) d \mu_{k}(x) d \mu_{k}(y),
$$

for $u, v \in \mathcal{C}_{k}$, where $\left\{\mu_{k}\right\}$ stands for a sequence of Radon measures with a vague limit $\mu_{F}$. $\left\{F_{k}\right\}$ for a sequence of closed sets in $\boldsymbol{Z}_{p}$ and $\rho_{k}(x, y)$ for a non-negative locally constant function on $\boldsymbol{Z}_{p} \times \boldsymbol{Z}_{p} \backslash\left\{(x, x) ; x \in \boldsymbol{Z}_{p}\right\}$. In fact, particularly by choosing $\mu_{k}$ as an identical Radon measure, $\rho_{k}(x, y)$ as $K(x, y)$ and $F_{k}$ as the whole space $\boldsymbol{Z}_{p}$ independent of $k$, we can cover the case which was dealt with in [7].

A main reason to focus on such sequence of Dirichlet forms can be found in a construction of a random fractal. In fact, we take a positive real number $\alpha$ satisfying $\alpha<1-t_{2}$ with the real parameter $t_{2}$ and the sequence $\left\{\mu_{k}\right\}$ of random Radon measures in the article [10] and introduce a sequence of random symmetric bilinear forms $\left\{\mathcal{E}_{F_{k}}\right\}$ defined by

$$
\mathcal{E}_{F_{k}}(u, v)=\iint_{\left(F_{k} \times F_{k}\right) \cap\{x \neq y\}}(u(x)-u(y))(v(x)-v(y))\|x-y\|^{-\alpha} d \mu_{k}(x) d \mu_{k}(y),
$$

for locally constant functions $u, v$ on $\boldsymbol{Z}_{p}$, where $\|x-y\|$ stands for the $p$-adic norm of $x-y \in$ $\boldsymbol{Z}_{p}$. Hereafter, the family of locally constant function on $\boldsymbol{Z}_{p}$ will be denoted by $\mathcal{C}$ and the Haar measure on $\boldsymbol{Z}_{p}$ by $\mu$.

In the construction of the random fractal $F$ in [10], $F_{k}$ is obtained right after removal of a ball with radius $p^{-\ell_{k}}$ in [10], $F_{k}$ is described as a union of balls with radius $p^{-\ell_{k}}$. By recalling the fact that $\ell_{k}<k$ for sufficiently large $k$, we will regard $F_{k}$ as a union of balls with radius $p^{-(k+1)}$ to subdue the use of suffix in notations. We already saw in [10] that, for any ball $A$ in $\boldsymbol{Z}_{p},\left\{\mu_{k}(A)\right\}$ is an $\left\{\mathcal{F}_{k}\right\}$-martingale with the deterministic initial value given by the Haar measure $\mu(A)$ of $A$. In particular, we focused on a sequence $\left\{\mu_{k}\right\}_{k=1}^{\infty}$ of random Radon measures on $\boldsymbol{Z}_{p}$ defined by $\mu_{k}(A)=\left(\Pi_{j=1}^{k}\left(1-p^{-\ell_{j}}\right)^{-1}\right) \mu\left(A \cap F_{k}\right)$ and satisfying $E\left(\mu_{k}(A)\right)=\mu(A)$ for any ball $A$ in $\boldsymbol{Z}_{p}$. We obtained the random Radon measure $\mu_{F}$ on $\boldsymbol{Z}_{p}$ which is uniquely characterized by $\mu_{F}(A)=\lim _{k \rightarrow \infty} \mu_{k}(A)$ for any ball $A \subset \boldsymbol{Z}_{p}$ with probability one. In our compact space $\boldsymbol{Z}_{p}$, Radon measure has finite total mass and the finiteness of $\mu_{F}$ with probability one follows from $E\left(\mu_{F}\left(\boldsymbol{Z}_{p}\right)\right)=\mu\left(\boldsymbol{Z}_{p}\right)=1$ obtained in [10]. 
If the sequence $\left\{A_{n}\right\}$ of the balls each of which is taken for the $n$-th cut out procedure satisfies $\liminf _{k \rightarrow \infty} k \mu\left(A_{k}\right)=t_{1}$ and $\limsup _{k \rightarrow \infty} k \mu\left(A_{k}\right)=t_{2}$ for some $t_{1}$ and $t_{2}$ with

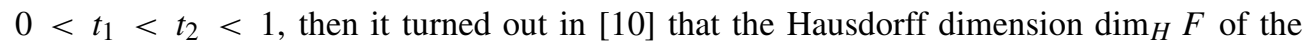
random fractal $F$ given by $F=\cap_{k=1}^{\infty} A_{k}^{c}$ satisfies $\operatorname{dim}_{H} F \leq 1-t_{1}$ with probability one and $\operatorname{dim}_{H} F \geq 1-t_{2}$ with a positive probability. To be more precise, $\mu_{F}$ concentrates only on $F$ and

$$
\int_{F} \int_{F} \frac{1}{\|x-y\|^{\alpha}} d \mu_{F}(x) d \mu_{F}(y)<\infty
$$

holds valid with probability one for any $\alpha$ satisfying $0<\alpha<1-t_{2}$ and $\mu_{F}(F)>0$ with a positive probability. Therefore, we can introduce the bilinear form

$$
\mathcal{E}_{F}(u, v)=\iint_{(F \times F) \cap\{x \neq y\}}(u(x)-u(y))(v(x)-v(y))\|x-y\|^{-\alpha} d \mu_{F}(x) d \mu_{F}(y),
$$

for any pair of functions $u, v$ in $\mathcal{C}$ with probability one.

PROPOSITION 1.1. The sequence $\left\{\mathcal{E}_{F_{k}}\right\}$ of symmetric bilinear forms converges to $\mathcal{E}_{F}$, in the sense that

$$
\lim _{k \rightarrow \infty} \mathcal{E}_{F_{k}}(u, v)=\mathcal{E}_{F}(u, v),
$$

for any pair of functions $u, v$ in $\mathcal{C}$ with probability one.

Proof. As pointed out in Chapter VII Lemma 1 in [18], any function in $\mathcal{C}$ is described as $\sum_{i=1}^{n} a_{i} 1_{B_{i}}$ with some real coefficients $a_{1}, \ldots, a_{n}$, disjoint balls $B_{1}, \ldots, B_{n}$ in $\boldsymbol{Z}_{p}$ and positive integer $n$. It suffices to show the convergence of the sequence $\left\{\mu_{k}(A) \mu_{k}(B)\right\}$ to $\mu_{F}(A) \mu_{F}(B)$ for any disjoint balls $A, B$ in $Z_{p}$ with probability one. The convergence follows from the vague convergence of $\left\{\mu_{k}\right\}$ to $\mu_{F}$ with probability one.

Thanks to these observations, we may concentrate our interest to the case that a nonrandom Radon measure $\mu_{F}$ satisfying

$$
\lim _{k \rightarrow \infty} \mathcal{E}_{F_{k}}(u, v)=\mathcal{E}_{F}(u, v)
$$

for any pair of functions $u, v$ in $\mathcal{C}$ is given as the vague limit of non-random Radon measures $\left\{\mu_{k}\right\}$.

Since $\left\{\mathcal{E}_{F_{k}}\right\}$ is represented as $\left\{\mathcal{E}_{k}\right\}$ by taking the function $1 /\|x-y\|^{\alpha}$ as $\rho_{k}(x, y)$ independently chosen in $k$, we may concentrate our attention only on the sequence $\left\{\mathcal{E}_{k}\right\}$ represented by (1). By denoting either the Haar measure used in [7] or $\mu_{F}$ introduced in [10] by $\mu_{0}$ in either case discussed so far, the bilinear forms obtained as the limit are written by a single representation

$$
\mathcal{E}_{0}(u, v)=\frac{1}{2} \iint_{\left(Z_{p} \times Z_{p}\right) \cap\{x \neq y\}}(u(x)-u(y))(v(x)-v(y)) \rho(x, y) \mu_{0}(d x) \mu_{0}(d y)
$$

for any pair of functions $u, v$ in $\mathcal{C}$.

Similarly to [8], for any sequence $\{c(m)\}_{m \in \boldsymbol{Z}_{-} \cup\{0\}}$ with $\boldsymbol{Z}_{-}=\{m \in \boldsymbol{Z} ; m<0\}$ satisfying $c(0) \leq c(-1) \leq \cdots \leq c(m) \leq \cdots$ and Radon measure $v$ on $\boldsymbol{Z}_{p}$, we can define 
$\bar{b}_{x, m}^{(\nu)}=v\left(B\left(x, p^{m}\right)\right)$ and $b_{x, \ell}^{(v)}=v\left(B\left(x, p^{\ell}\right) \backslash B\left(x, p^{\ell-1}\right)\right)$ non-positive integers $m, \ell$ and take a symmetric bilinear form

$$
\mathcal{E}^{(\{c\}, v)}(u, v)=\iint_{\left(\boldsymbol{Z}_{p} \times \boldsymbol{Z}_{p}\right) \cap\{x \neq y\}}(u(x)-u(y))(v(x)-v(y)) \rho^{(\{c\}, v)}(x, y) d v(x) d v(y)
$$

where $\rho^{(\{c\}, v)}(x, y)=\sum_{\ell=m+1}^{0} b_{x, \ell}^{(v)} c(\ell)(2 c(m)-c(\ell))-\bar{b}_{x, m}^{(v)} c(m)^{2}$ with the integer $m$ satisfying $p^{m}=\|x-y\|$.

For the sequence $\left\{\mu_{k}\right\}$ of the Radon measure arising from the random fractal and its vague limit $\mu_{0}$, we obtain the sequence $\left\{\mathcal{E}^{\left(\{c\}, \mu_{k}\right)}\right\}$ of the symmetric bilinear forms and $\left\{\mathcal{E}^{\left(\{c\}, \mu_{0}\right)}\right\}$. As a corollary of Proposition 1.1, we have the following assertion:

COROLlaRY 1.2. The sequence $\left\{\mathcal{E}^{\left(\{c\}, \mu_{k}\right)}\right\}$ converges to $\mathcal{E}^{\left(\{c\}, \mu_{0}\right)}$, in the sense that

$$
\lim _{k \rightarrow \infty} \mathcal{E}^{\left(\{c\}, \mu_{k}\right)}(u, v)=\mathcal{E}^{\left(\{c\}, \mu_{0}\right)}(u, v)
$$

for any pair of functions $u, v$ in $\mathcal{C}$ with probability one.

Proof. We may assume that $u=1_{B}$ and $v=1_{B^{\prime}}$ for some distinct balls $B, B^{\prime}$ with radius $p^{-\ell}$. Since $\rho^{\left(\{c\}, \mu_{k}\right)}(x, y)$ is independent of $\left(x, x^{\prime}\right)$ with $x \in B$ and $x^{\prime} \in B^{\prime}$, the value can be represented by $\rho^{\left(\{c\}, \mu_{k}\right)}\left(B, B^{\prime}\right)$. By denoting the distance between $B$ and $B^{\prime}$ by $p^{-m}$, the vague convergence of $\left\{\mu_{k}\right\}$ to $\mu$ shows that $\rho^{\left(\{c\}, \mu_{k}\right)}\left(B, B^{\prime}\right)=\sum_{\nu=m+1}^{0} b_{x, v}^{\left(\mu_{k}\right)} c(v)(2 c(m)-$ $c(v))-\bar{b}_{x, m}^{\left(\mu_{0}\right)} c(m)^{2}$ tends to $\rho^{\left(\{c\}, \mu_{0}\right)}\left(B, B^{\prime}\right)=\sum_{\nu=m+1}^{0} b_{x, \nu}^{\left(\mu_{0}\right)} c(v)(2 c(m)-c(v))-\bar{b}_{x, m}^{\left(\mu_{0}\right)} c(m)^{2}$ as $k \rightarrow \infty$. Therefore, by combining this with the convergence of $\left\{\mu_{k}(B) \mu_{k}\left(B^{\prime}\right)\right\}$ shown in the proof of Proposition 1.1, we have $\lim _{k \rightarrow \infty} \mathcal{E}^{\left(\{c\}, \mu_{k}\right)}\left(1_{B}, 1_{B^{\prime}}\right)=\lim _{k \rightarrow \infty} \rho^{\left(\{c\}, \mu_{k}\right)}\left(B, B^{\prime}\right)$ $\mu_{k}(B) \mu_{k}\left(B^{\prime}\right)=\rho^{\left(\{c\}, \mu_{0}\right)}\left(B, B^{\prime}\right) \mu_{0}(B) \mu_{0}\left(B^{\prime}\right)=\mathcal{E}^{\left(\{c\}, \mu_{0}\right)}\left(1_{B}, 1_{B^{\prime}}\right)$.

This corollary allows us to choose $\rho^{\left(\{c\}, \mu_{k}\right)}$ as $\rho_{k}$ in (1) for regarding $\mathcal{E}^{\left(\{c\}, \mu_{k}\right)}$ as $\mathcal{E}_{k}$ and choose $\rho^{\left(\{c\}, \mu_{0}\right)}$ as $\rho$ in (2) for regarding $\mathcal{E}^{\left(\{c\}, \mu_{0}\right)}$ as $\mathcal{E}_{0}$. In what follows, we will establish a method to address the convergence of Hunt processes relevant to the sequences $\left\{\mathcal{E}_{H_{k}, Z_{p}}\right\},\left\{\mathcal{E}_{F_{k}}\right\}$ and $\left\{\mathcal{E}^{\left(\{c\}, \mu_{k}\right)}\right\}$ of Dirichlet forms each of which converges to $\mathcal{E}_{K}, \mathcal{E}_{F}$ and $\mathcal{E}^{\left(\{c\}, \mu_{0}\right)}$ respectively as symmetric bilinear forms. From now on, whichever sequence of Dirichlet forms is addressed, we will denote the sequence of Dirichlet forms by $\left\{\mathcal{E}_{k}\right\}$ and its limit by $\mathcal{E}_{0}$. In building our scheme, we may concentrate our focus on the case that $\rho$ and any $\rho_{k}$ in the sequence take constant on every Cartesian product $B \times B^{\prime}$ of distinct balls $B$ and $B^{\prime}$ with the same radius in $\boldsymbol{Z}_{p}$ and that $\lim _{k \rightarrow \infty} \rho_{k}(x, y)=\rho(x, y)$ for any pair $x, y$ of distinct points in $\boldsymbol{Z}_{p}$.

LEMMA 1.3. The symmetric bilinear form $\left(\mathcal{E}_{0}, \mathcal{C}\right)$ is closable on $L^{2}\left(\boldsymbol{Z}_{p} ; \mu_{0}\right)$.

PROOF. We assume that a sequence $\left\{u_{n}\right\}$ in $\mathcal{C}$ satisfies $\lim _{n, m \rightarrow \infty} \mathcal{E}_{0}\left(u_{n}-u_{m}, u_{n}-\right.$ $\left.u_{m}\right)=0$ and $\lim _{n \rightarrow \infty}\left\|u_{n}\right\|_{L^{2}\left(Z_{p} ; \mu_{0}\right)}=0$. These conditions imply that, for any $\varepsilon>0$, there exists a subsequence $\left\{u_{n^{\prime}}\right\}$ satisfying $\mathcal{E}_{0}\left(u_{n^{\prime}}-u_{m^{\prime}}, u_{n^{\prime}}-u_{m^{\prime}}\right) \leq \varepsilon$ and $\lim _{n^{\prime} \rightarrow \infty} u_{n^{\prime}}(x)=0$ almost every $x$ in the support of the measure $\mu_{0}$. Then, one sees 


$$
\begin{aligned}
& \mathcal{E}_{0}\left(u_{n^{\prime}}, u_{n^{\prime}}\right) \\
& \quad \leq \liminf _{m^{\prime} \rightarrow \infty} \iint_{\left(\mathbf{Z}_{p} \times \mathbf{Z}_{p}\right) \cap\{x \neq y\}}\left(u_{n^{\prime}}(x)-u_{m^{\prime}}(x)-u_{n^{\prime}}(y)-u_{m^{\prime}}(y)\right)^{2} \rho(x, y) d \mu_{0}(x) d \mu_{0}(y) \\
& \quad \leq \varepsilon .
\end{aligned}
$$

By repeating the procedure of taking subsequence, one can take some subsequence $\left\{u_{m}\right\}$ of $\left\{u_{n}\right\}, \lim _{m \rightarrow \infty} \mathcal{E}_{0}\left(u_{m}, u_{m}\right)=0$. This implies $\lim _{n \rightarrow \infty} \mathcal{E}_{0}\left(u_{n}, u_{n}\right)=0$.

This lemma shows that $\mathcal{E}_{0}$ admits the symmetric bilinear form $\mathcal{E}$ with the domain $\mathcal{F}$ obtained as the smallest closed extension of $\mathcal{E}_{0}$ and as its domain.

2. Lemmas. For any $\delta>0$, we define the symmetric bilinear form

$$
\mathcal{E}_{k}^{(\delta)}(u, v)=\iint_{\left(\boldsymbol{Z}_{p} \times \boldsymbol{Z}_{p}\right) \cap\{\|x-y\|>\delta\}}(u(x)-u(y))(v(x)-v(y)) \rho_{k}(x, y) d \mu_{k}(x) d \mu_{k}(y)
$$

with domain $\mathcal{C}_{k}$ and

$$
\mathcal{E}^{(\delta)}(u, u)=\iint_{\left(\boldsymbol{Z}_{p} \times \boldsymbol{Z}_{p}\right) \cap\{\|x-y\|>\delta\}}(u(x)-u(y))(v(x)-v(y)) \rho(x, y) d \mu_{0}(x) d \mu_{0}(y)
$$

with domain $\mathcal{C}$. Then, $\mathcal{E}_{k}$ and $\mathcal{E}$ admit similar expressions obtained by taking $\delta=0$.

In discussing the convergence of $L^{2}\left(\boldsymbol{Z}_{p} ; \mu_{k}\right)$ to $L^{2}\left(\boldsymbol{Z}_{p} ; \mu_{0}\right)$ in the sense of Kuwae and Shioya [15], we can take the identity map as the linear operator $\Phi_{k}: \mathcal{C} \rightarrow L^{2}\left(\boldsymbol{Z}_{p} ; \mu_{k}\right)$ for any $k$. This is because the vague convergence of $\left\{\mu_{k}\right\}$ to $\mu_{0} \operatorname{implies} \lim _{k \rightarrow \infty}\|u\|_{L^{2}\left(Z_{p} ; \mu_{k}\right)}=$ $\|u\|_{L^{2}\left(Z_{p} ; \mu_{0}\right)}$ for any $u \in \mathcal{C}$.

For our situation, we can restate the definition of the KS-strongly convergence in [15] as follows: A sequence $\left\{v_{k}\right\}_{k=1}^{\infty}$ of functions $v_{k} \in L^{2}\left(\boldsymbol{Z}_{p} ; \mu_{k}\right)$ is said to be KS-strongly convergent to a function $v \in L^{2}\left(\boldsymbol{Z}_{p} ; \mu_{0}\right)$, if there exists a sequence $\left\{\varphi_{j}\right\}_{j=1}^{\infty} \subset \mathcal{C}$ such that $\lim _{j \rightarrow \infty} \limsup _{k \rightarrow \infty}\left\|\varphi_{j}-v_{k}\right\|_{L^{2}\left(\boldsymbol{Z}_{p} ; \mu_{k}\right)}=0$ and $\lim _{j \rightarrow \infty}\left\|\varphi_{j}-v\right\|_{L^{2}\left(\boldsymbol{Z}_{p} ; \mu_{0}\right)}=0$. Then we recall that a sequence $\left\{u_{k}\right\}_{k=1}^{\infty}$ of functions $u_{k} \in L^{2}\left(\boldsymbol{Z}_{p} ; \mu_{k}\right)$ is said to be KS-weakly convergent to a function $u \in L^{2}\left(\boldsymbol{Z}_{p} ; \mu_{0}\right)$, if $\lim _{k \rightarrow \infty}\left(u_{k}, v_{k}\right)_{L^{2}\left(\boldsymbol{Z}_{p} ; \mu_{k}\right)}=(u, v)_{L^{2}\left(\boldsymbol{Z}_{p} ; \mu_{0}\right)}$ for any KS-strongly convergent sequence $\left\{v_{k}\right\}_{k=1}^{\infty}$ with $v_{k} \in L^{2}\left(\boldsymbol{Z}_{p} ; \mu_{k}\right)$ and the KS-strong limit $v$.

Let us take a KS-weakly convergent sequence $\left\{u_{k}\right\}_{k=1}^{\infty}$ with a KS-weak limit in $L^{2}\left(\boldsymbol{Z}_{p}\right.$; $\left.\mu_{0}\right)$ and fix a positive integer $\ell$ satisfying $p^{-\ell} \leq \delta$. For a given locally integrable function $u$ on $\boldsymbol{Z}_{p}$ with respect to $\mu_{k}$, we define a locally constant function on $\boldsymbol{Z}_{p}$ by

$$
(u)_{\mu_{k}, \ell}(x)=\frac{1}{\mu_{k}\left(B\left(x, p^{-\ell}\right)\right)} \int_{B\left(x, p^{-\ell}\right)} u(y) d \mu_{k}(y) .
$$

LEMMA 2.1. (i) $\sup _{k}\left\|u_{k}\right\|_{L^{2}\left(\boldsymbol{Z}_{p} ; \mu_{k}\right)}<\infty$,

(ii) $k \geq \ell$ implies $\int_{\boldsymbol{Z}_{p}}\left(u_{k}\right)_{\mu_{k}, \ell}(x) d \mu_{k}(x)=\int_{\boldsymbol{Z}_{p}} u_{k}(x) d \mu_{k}(x)$,

(iii) $\sup _{\ell} \sup _{k \geq \ell}\left\|\left(u_{k}\right)_{\mu_{k}, \ell}\right\|_{L^{2}\left(Z_{p} ; \mu_{k}\right)}<\infty$,

(iv) there exists a sequence $\{K(\ell)\}_{\ell=1}^{\infty}$ of non-negative integers such that $\sup _{\ell} \sup _{k \geq K(\ell)}$ $\left\|\left(u_{k}\right)_{\mu_{k}, \ell}\right\|_{L^{2}\left(Z_{p} ; \mu_{0}\right)}<\infty$. 
PROOF. (i) The assertion follows from Lemma 2.3 in [15].

(ii) The identity is verified by the identities

$$
\begin{aligned}
\int_{Z_{p}}(u)_{\mu_{k}, \ell}(x) d \mu_{k}(x) & =\int_{Z_{p}} \frac{1}{\mu_{k}\left(B\left(x, p^{-\ell}\right)\right)} \int_{B\left(x, p^{-\ell}\right)} u(y) d \mu_{k}(y) d \mu_{k}(x) \\
& =\int_{Z_{p}} \frac{1}{\mu_{k}\left(B\left(x, p^{-\ell}\right)\right)} \int_{Z_{p}} u(y) 1_{B\left(y, p^{-\ell}\right)}(x) d \mu_{k}(y) d \mu_{k}(x) \\
& =\int_{Z_{p}} \int_{Z_{p}} \frac{1}{\mu_{k}\left(B\left(x, p^{-\ell}\right)\right)} u(y) 1_{B\left(y, p^{-\ell}\right)}(x) d \mu_{k}(y) d \mu_{k}(x) \\
& =\int_{Z_{p}} \frac{\mu_{k}\left(B\left(y, p^{-\ell}\right)\right)}{\mu_{k}\left(B\left(y, p^{-\ell}\right)\right)} u(y) d \mu_{k}(y) \\
& =\int_{Z_{p}} u(x) d \mu_{k}(x)
\end{aligned}
$$

for any measurable function $u$ on $Z_{p}$ with $\operatorname{supp}[u] \subset F_{k}$, where we use the identity, $B\left(x, p^{-\ell}\right)=B\left(y, p^{-\ell}\right)$ for any $k \geq \ell$.

(iii) Since $(u)_{\mu_{k}, \ell}(x)^{2} \leq\left(u^{2}\right)_{\mu_{k}, \ell}(x)$ on $Z_{p}$ as well for any measurable function $u$ on $\boldsymbol{Z}_{p}$, the finiteness of $\sup _{\ell} \sup _{k \geq \ell}\left\|\left(u_{k}\right)_{\mu_{k}, \ell}\right\|_{L^{2}\left(\boldsymbol{Z}_{p} ; \mu_{k}\right)}$ can be verified by (i) and (ii).

(iv) The sequence of the non-negative integers in the assertion is obtained by (iii) and the inequality

$$
\int_{\boldsymbol{Z}_{p}}|v(x)|^{2} d \mu_{k}(x) \geq \frac{1}{2} \int_{\boldsymbol{Z}_{p}}|v(x)|^{2} d \mu_{0}(x)
$$

valid for any function $v$ on $\boldsymbol{Z}_{p}$ taking constant on every ball with radius $p^{-\ell}$ and sufficiently large $k$.

For any pair $B, B^{\prime}$ of disjoint balls with the same radius $p^{-\ell}$ with $p^{-\ell} \leq \delta$, we denote the values $\rho\left(x, x^{\prime}\right)$ and $\rho_{k}\left(x, x^{\prime}\right)$ determined independently of the choice $x \in B, x^{\prime} \in B^{\prime}$ by $\rho\left(B, B^{\prime}\right)$ and by $\rho_{k}\left(B, B^{\prime}\right)$, respectively, and then we see $\rho_{k}\left(B, B^{\prime}\right) \geq(1-\delta) \rho\left(B, B^{\prime}\right)$ for sufficiently large $k$ owing to the convergence $\lim _{k \rightarrow \infty} \rho_{k}(x, y)=\rho(x, y)$ for any distinct $x, y \in Z_{p}$.

Since one can observe that

$$
\begin{aligned}
\mid(u)_{\mu_{k}, \ell} & (x)-(u)_{\mu_{k}, \ell}(y) \mid \\
= & \frac{1}{\mu_{k}\left(B\left(x, p^{-\ell}\right)\right) \mu_{k}\left(B\left(y, p^{-\ell}\right)\right)} \\
& \times\left|\int_{B\left(x, p^{-\ell}\right)} \int_{B\left(y, p^{-\ell}\right)}(u(w)-u(z)) d \mu_{k}(w) d \mu_{k}(z)\right| \\
\leq & \left(\frac{1}{\mu_{k}\left(B\left(x, p^{-\ell}\right)\right) \mu_{k}\left(B\left(y, p^{-\ell}\right)\right)}\right.
\end{aligned}
$$




$$
\left.\times \int_{B\left(x, p^{-\ell}\right)} \int_{B\left(y, p^{-\ell}\right)}(u(w)-u(z))^{2} d \mu_{k}(w) d \mu_{k}(z)\right)^{1 / 2}
$$

with the right-hand side independent of $x \in B, y \in B^{\prime}$ for any balls $B, B^{\prime}$ with the radius $p^{-\ell}$ and that $\mu_{k}(B) \geq(1-\delta) \mu_{0}(B)$ for any ball $B$ with the radius $p^{-\ell}$ and sufficiently large $k$,

$$
\begin{aligned}
\mathcal{E}_{k}(u, u) \geq & \mathcal{E}_{k}^{(\delta)}(u, u) \\
\geq & \iint \frac{1_{\{\|x-y\|>\delta\}}}{\mu_{k}\left(B\left(x, p^{-\ell}\right)\right) \mu_{k}\left(B\left(y, p^{-\ell}\right)\right)} \\
& \times \int_{B\left(x, p^{-\ell}\right)} \int_{B\left(y, p^{-\ell}\right)}(u(w)-u(z))^{2} \rho_{k}(z, w) d \mu_{k}(w) d \mu_{k}(z) d \mu_{k}(x) d \mu_{k}(y) \\
\geq & \iint_{\{\|x-y\|>\delta\}}\left((u)_{\mu_{k}, \ell}(x)-(u)_{\mu_{k}, \ell}(y)\right)^{2} \rho_{k}(x, y) d \mu_{k}(x) d \mu_{k}(y) \\
= & \sum_{B \cap B^{\prime} \neq \emptyset, \operatorname{diam}(B)=\operatorname{diam}\left(B^{\prime}\right)=p^{-\ell}}\left((u)_{\mu_{k}, \ell}(B)-(u)_{\mu_{k}, \ell}\left(B^{\prime}\right)\right)^{2} \rho_{k}\left(B, B^{\prime}\right) \mu_{k}(B) d \mu_{k}\left(B^{\prime}\right) \\
\geq & (1-\delta)^{3} \sum_{B \cap B^{\prime} \neq \emptyset}\left((u)_{\mu_{k}, \ell}(B)-(u)_{\mu_{k}, \ell}\left(B^{\prime}\right)\right)^{2} \rho\left(B, B^{\prime}\right) \mu_{0}(B) d \mu_{0}\left(B^{\prime}\right) \\
\geq & (1-\delta)^{3} \iint_{\{\|x-y\|>\delta\}}\left((u)_{\mu_{k}, \ell}(x)-(u)_{\mu_{k}, \ell}(y)\right)^{2} \rho(x, y) d \mu_{0}(x) d \mu_{0}(y),
\end{aligned}
$$

where $(u)_{\mu_{k}, \ell}(B)$ and $(u)_{\mu_{k}, \ell}\left(B^{\prime}\right)$ stand for the values $(u)_{\mu_{k}, \ell}(x)$ and $(u)_{\mu_{k}, \ell}(y)$ determined independently of the choice $x \in B, y \in B^{\prime}$ respectively.

For any KS-weakly convergent sequence $\left\{u_{k}\right\}_{k=1}^{\infty}$, by taking sufficiently large $k$, we have

$$
\begin{aligned}
\mathcal{E}_{k}\left(u_{k}, u_{k}\right) \geq & \mathcal{E}_{k}^{(\delta)}\left(u_{k}, u_{k}\right) \\
\geq & \iint \frac{1_{\{\|x-y\|>\delta\}}}{\mu_{k}\left(B\left(x, p^{-\ell}\right)\right) \mu_{k}\left(B\left(y, p^{-\ell}\right)\right)} \\
& \times \int_{B\left(x, p^{-\ell}\right)} \int_{B\left(y, p^{-\ell}\right)}\left(u_{k}(w)-u_{k}(z)\right)^{2} \rho_{k}(z, w) d \mu_{k}(w) d \mu_{k}(z) d \mu_{k}(x) d \mu_{k}(y) \\
\geq & (1-\delta)^{3} \iint_{\{\|x-y\|>\delta\}}\left(\left(u_{k}\right)_{\mu_{k}, \ell}(x)-\left(u_{k}\right)_{\mu_{k}, \ell}(y)\right)^{2} \rho(x, y) d \mu_{0}(x) d \mu_{0}(y) \\
\geq & (1-\delta)^{3} \mathcal{E}^{(\delta)}\left(\left(u_{k}\right)_{\mu_{k}, \ell},\left(u_{k}\right)_{\mu_{k}, \ell}\right) .
\end{aligned}
$$

We may assume that $\sup _{k} \mathcal{E}_{k}\left(u_{k}, u_{k}\right)<\infty$ without losing general setting in our discussion on the generalized Mosco-convergence. Accordingly, the boundedness of the sequence $\left\{\left(u_{k}\right)_{\mu_{k}, \ell}\right\}_{k \geq K(\ell)}$ with respect to the norm $\left(\mathcal{E}^{(\delta)}(u, u)+\|u\|_{L^{2}\left(Z_{p} ; \mu_{0}\right)}^{2}\right)^{1 / 2}$ is obtained.

In dealing with the measure $\mu_{0}$, we may remove the balls with radius $p^{-\ell}$ from $Z_{p}$ which are not charged by the measure $\mu_{0}$. For a precise description, we denote the family of all balls with radius $p^{-\ell}$ contained in $Z_{p}$ by $\left\{B_{i} ; i=0, \ldots, p^{\ell}-1\right\}$ and replace $Z_{p}$ with $S_{\ell}=$ $\bigcup_{i \in I_{\ell}} B_{i}$ by introducing $I_{\ell}=\left\{i \in\left\{0, \ldots, p^{\ell}-1\right\} ; \mu_{0}\left(B_{i}\right)>0\right\}$ in the definition of the KSstrong convergence. For instance, we can observe the KS-strong convergence of $\left\{v_{k} 1_{S_{\ell}}\right\}_{k=0}^{\infty}$ 
to $v 1_{S_{\ell}}$ when the KS-strong convergence of the sequence $\left\{v_{k}\right\}_{k=0}^{\infty}$ to $v$ is obtained. Here, we introduce a positive finitely-many valued function $\left\{g_{\ell}\right\}$ and a sequence $\left\{h_{k, \ell}\right\}$ of finitely-many valued functions on $S_{\ell}$ by $g_{\ell}(x)=\sum_{i \in I_{\ell}} \mu_{0}\left(B_{i}\right) 1_{B_{i}}(x)$ and $h_{k, \ell}(x)=\sum_{i \in I_{\ell}} \mu_{k}\left(B_{i}\right) 1_{B_{i}}(x)$. Then the vague convergence of $\left\{\mu_{k}\right\}_{k=1}^{\infty}$ to $\mu_{0}$ implies $\lim _{k \rightarrow \infty} h_{k, \ell}(x)=g_{\ell}(x)$ at each $x \in$ $S_{\ell}$. In what follows, we will denote $\operatorname{supp}\left[\mu_{k}\right]$ by $F_{k}$ and $\operatorname{supp}\left[\mu_{0}\right]$ by $F$. Now we are in position to discuss the following convergences:

LEMMA 2.2. For any $K S$-weakly convergent sequence $\left\{u_{k}\right\}_{k=1}^{\infty}$ with the $K S$-weak limit $u \in L^{2}\left(F ; \mu_{0}\right)$,

(i) $\left\{u_{k}\left(g_{\ell} / h_{k, \ell}\right) 1_{S_{\ell}}\right\}_{k=1}^{\infty}$ is a $K S$-weakly convergent sequence to $u$,

(ii) there exists a subsequence $\left\{k_{\ell}\right\}$ satisfying $\lim _{\ell \rightarrow \infty}\left(\left(u_{k_{\ell}} 1_{S_{\ell}}\right)_{k_{\ell}, \ell}, 1_{B}\right)_{L^{2}\left(F_{k_{\ell}} ; \mu_{k_{\ell}}\right)}=$ $\left(u, 1_{B}\right)_{L^{2}\left(F ; \mu_{0}\right)}$ for any ball $B$ in $\boldsymbol{Z}_{p}$.

PROOF. (i) Since the KS-strong convergence of $\left\{v_{k}\right\}_{k=1}^{\infty}$ to $v$ implies the KS-strong convergence of $\left\{v_{k}\left(g_{\ell} / h_{k, \ell}\right) 1_{S_{\ell}}\right\}_{k=1}^{\infty}$ to $v 1_{S_{\ell}}$, we obtain the KS-weak convergence of $\left\{u_{k}\left(g_{\ell} / h_{k, \ell}\right)\right.$ $\left.1_{S_{\ell}}\right\}_{k=1}^{\infty}$ to $u$ by the identities

$$
\begin{aligned}
\lim _{k \rightarrow \infty}\left(u_{k} \frac{g_{\ell}}{h_{k, \ell}} 1_{S_{\ell}}, v_{k}\right)_{L^{2}\left(F_{k} ; \mu_{k}\right)} & =\lim _{k \rightarrow \infty}\left(u_{k}, v_{k} \frac{g_{\ell}}{h_{k, \ell}} 1_{S_{\ell}}\right)_{L^{2}\left(F_{k} ; \mu_{k}\right)} \\
& =\left(u, v 1_{\left.S_{\ell}\right)_{L^{2}}\left(F ; \mu_{0}\right)}\right. \\
& =(u, v)_{L^{2}\left(F ; \mu_{0}\right)}
\end{aligned}
$$

for any KS-strong convergent sequence $\left\{v_{k}\right\}_{k=1}^{\infty}$ to $v$.

(ii) Thanks to (i), we see $\lim _{k \rightarrow \infty}\left(u_{k}\left(g_{\ell} / h_{k, \ell}\right) 1_{S_{\ell}}, v_{k}\right)_{L^{2}\left(F_{k} ; \mu_{k}\right)}=(u, v)_{L^{2}\left(F ; \mu_{0}\right)}$ for any $\ell$ and any KS-strong convergent sequence $\left\{v_{k}\right\}_{k=1}^{\infty}$ to $v$. Due to the KS-strong convergence of $\left\{1_{B}\right\}_{k=1}^{\infty}$ to $1_{B}$ for any ball $B$ in $Z_{p}$, we can take a subsequence $\left\{k_{\ell}\right\}$ of $k$ satisfying

$$
\lim _{\ell \rightarrow \infty}\left(u_{k_{\ell}} \frac{g_{\ell}}{h_{k_{\ell}, \ell}} 1_{S_{\ell}}, 1_{B}\right)_{L^{2}\left(F_{k_{\ell}} ; \mu_{k_{\ell}}\right)}=\left(u, 1_{B}\right)_{L^{2}\left(F ; \mu_{0}\right)}
$$

by an ordinary method for doubly indexed sequence. The assertion follows from the following identities:

$$
\begin{aligned}
& \left(\left(u_{k_{\ell}} 1_{S_{\ell}}\right)_{\mu_{k}, \ell}, 1_{B}\right)_{L^{2}\left(F ; \mu_{0}\right)} \\
& \quad=\int_{Z_{p}} \frac{1}{\mu_{k_{\ell}}\left(B\left(x, p^{-\ell}\right) \cap F_{k_{\ell}}\right)} \int_{B\left(x, p^{-\ell}\right) \cap F_{k_{\ell}}} u_{k_{\ell}}(y) 1_{S_{\ell}}(y) d \mu_{k_{\ell}}(y) 1_{B}(x) d \mu_{0}(x) \\
& \quad=\int_{Z_{p}} \frac{1}{\mu_{k_{\ell}}\left(B\left(x, p^{-\ell}\right) \cap F_{k_{\ell}}\right)} \int_{S_{\ell}} u_{k_{\ell}}(y) 1_{B\left(x, p^{-\ell}\right) \cap F_{k_{\ell}}}(y) d \mu_{k_{\ell}}(y) 1_{B}(x) d \mu_{0}(x) \\
& =\int_{Z_{p}} \frac{1}{\mu_{k_{\ell}}\left(B\left(x, p^{-\ell}\right) \cap F_{k_{\ell}}\right)} \int_{S_{\ell}} u_{k_{\ell}}(y) 1_{B\left(x, p^{-\ell}\right)}(y) 1_{F_{k_{\ell}}}(y) d \mu_{k_{\ell}}(y) 1_{B}(x) d \mu_{0}(x) \\
& =\int_{Z_{p}} \frac{1}{\mu_{k_{\ell}}\left(B\left(x, p^{-\ell}\right) \cap F_{k_{\ell}}\right)} \int_{S_{\ell}} u_{k_{\ell}}(y) 1_{B\left(y, p^{-\ell}\right)}(x) 1_{F}(x) d \mu_{k_{\ell}}(y) 1_{B}(x) d \mu_{0}(x)
\end{aligned}
$$




$$
\begin{aligned}
& =\int_{Z_{p}} \frac{1}{\mu_{k_{\ell}}\left(B\left(x, p^{-\ell}\right) \cap F_{k_{\ell}}\right)} \int_{S_{\ell}} u_{k_{\ell}}(y) 1_{B\left(y, p^{-\ell}\right)}(x) 1_{F}(x) d \mu_{k_{\ell}}(y) 1_{B}(y) d \mu_{0}(x) \\
& =\int_{Z_{p}} \frac{1}{\mu_{k_{\ell}}\left(B\left(x, p^{-\ell}\right) \cap F_{k_{\ell}}\right)} \int_{S_{\ell}} u_{k_{\ell}}(y) 1_{B\left(y, p^{-\ell}\right) \cap F}(x) d \mu_{k_{\ell}}(y) 1_{B}(y) d \mu_{0}(x) \\
& =\int_{Z_{p}} \int_{S_{\ell}} \frac{1}{\mu_{k_{\ell}}\left(B\left(y, p^{-\ell}\right) \cap F_{k_{\ell}}\right)} u_{k_{\ell}}(y) 1_{B\left(y, p^{-\ell}\right) \cap F}(x) d \mu_{k_{\ell}}(y) 1_{B}(y) d \mu_{0}(x) \\
& =\int_{S_{\ell}} \frac{\mu_{0}\left(B\left(y, p^{-\ell}\right) \cap F\right)}{\mu_{k_{\ell}}\left(B\left(y, p^{-\ell}\right) \cap F_{k_{\ell}}\right)} u_{k_{\ell}}(y) 1_{B}(y) d \mu_{k_{\ell}}(y) \\
& =\left(u_{k_{\ell}} \frac{g_{\ell}}{h_{k_{\ell}, \ell}} 1_{S_{\ell}, 1_{B}}\right)_{L^{2}\left(F_{k_{\ell}} ; \mu_{k_{\ell}}\right)}
\end{aligned}
$$

for sufficiently large $\ell$. This is due to $\operatorname{supp}\left[\mu_{\mathrm{k}_{\ell}}\right]=F_{k_{\ell}}$, $\operatorname{supp}\left[\mu_{0}\right]=F$ and the equivalence of $x \in B$ and $y \in B$ under the condition $\|x-y\| \leq p^{-\ell}$ for sufficiently large $\ell$.

Let $\left\{u_{k}\right\}$ be a KS-weakly convergent sequence with a KS-weak limit $u \in L^{2}\left(F ; \mu_{0}\right)$ satisfying $u_{k} \in \mathcal{C}_{k}$ for any $k=1,2, \ldots$ By applying the Lemma 2.2 (ii), we can take a subsequence $\left\{k_{\ell}\right\}$ of $\{k\}$ such that

$$
\begin{aligned}
\lim _{\ell \rightarrow \infty}\left(\left(u_{k_{\ell}}\right)_{\mu_{k_{\ell}}, \ell}, 1_{B}\right)_{L^{2}\left(F ; \mu_{0}\right)} & =\lim _{\ell \rightarrow \infty}\left(\left(u_{k_{\ell}} 1_{S_{\ell}}\right)_{\mu_{k_{\ell}}, \ell}, 1_{B}\right)_{L^{2}\left(F ; \mu_{0}\right)} \\
& =\left(u, 1_{B}\right)_{L^{2}\left(F ; \mu_{0}\right)}
\end{aligned}
$$

for any ball $B$ with sufficiently small radius, where the first identity follows from the identity $\left(u_{k_{\ell}}\right)_{\mu_{k_{\ell}}, \ell}=\left(u_{k_{\ell}} 1_{S_{\ell}}\right)_{\mu_{k_{\ell}}, \ell}$ on $S_{\ell}$ and $\operatorname{supp}\left[\mu_{0}\right]=F \subset S_{\ell}$ for any $\ell$.

3. Convergence of Hunt processes. In this section, we will validate the conditions (a) and (b) listed in Definition 2.1 (iv) of [6]. For that purpose, we consider the KS-weakly convergent sequence $\left\{u_{k}\right\}$ with the KS-weak limit $u$ and its subsequence $\left\{\left(u_{k_{\ell}}\right)_{\mu_{k}, \ell}\right\}$ taken in the previous section. We may assume that $\left\{\left(u_{k_{\ell}}\right)_{\mu_{k}, \ell}\right\}$ is extracted from $\left\{u_{k}\right\}$ so that $\left\{u_{k_{\ell}}\right\}$ satisfies $\lim _{\ell \rightarrow \infty} \mathcal{E}_{k_{\ell}}\left(u_{k_{\ell}}, u_{k_{\ell}}\right)=\liminf _{k \rightarrow \infty} \mathcal{E}_{k}\left(u_{k}, u_{k}\right)$. The Banach-Saks theorem shows that some subsequence $\left\{\left(u_{k_{\ell^{\prime}}}\right)_{\ell^{\prime}}\right\}$ of $\left\{\left(u_{k_{\ell}}\right)_{\mu_{k}, \ell}\right\}$ is extracted so as to converge weakly to some element $v$ in $L^{2}\left(F ; \mu_{0}\right)$ and satisfy

$$
\lim _{n \rightarrow \infty}\left\|\frac{1}{n} \sum_{\ell^{\prime}=1}^{n}\left(u_{k_{\ell^{\prime}}}\right)_{\ell^{\prime}}-v\right\|_{L^{2}\left(F ; \mu_{0}\right)}=0
$$

and

$$
\lim _{n \rightarrow \infty} \mathcal{E}^{(\delta)}\left(\frac{1}{n} \sum_{\ell^{\prime}=1}^{n}\left(u_{k_{\ell^{\prime}}}\right)_{\ell^{\prime}}-v, \frac{1}{n} \sum_{\ell^{\prime}=1}^{n}\left(u_{k_{\ell^{\prime}}}\right)_{\ell^{\prime}}-v\right)=0 .
$$

On the other hand, we have seen that $\lim _{\ell \rightarrow \infty}\left(\left(u_{k_{\ell}}\right)_{\mu_{k}, \ell}, 1_{B}\right)_{L^{2}\left(F ; \mu_{0}\right)}=\left(u, 1_{B}\right)_{L^{2}\left(F ; \mu_{0}\right)}$ for any ball $B$ with sufficiently small radius. Any $u \in L^{2}\left(F ; \mu_{0}\right)$ gives the functional $T(\varphi)=$ $\int_{F} u(x) \varphi(x) d \mu_{0}(x)$ defined for the restriction $\left.\varphi\right|_{F}$ of locally constant function $\varphi$ on $\boldsymbol{Z}_{p}$ to $F$ and the fact that $T$ vanishes if and only if $u$ is the zero element in $L^{2}\left(F ; \mu_{0}\right)$ implies the 
family $\mathcal{C}(F)$ of continuous functions on $F$ is a dense subfamily of $L^{2}\left(F ; \mu_{0}\right)$. Therefore, we conclude that $u=v$. Similarly to Section 5 in [6], we can prove

$$
\liminf _{\ell^{\prime} \rightarrow \infty} \mathcal{E}_{k_{\ell^{\prime}}}\left(u_{k_{\ell^{\prime}}}, u_{\ell_{\ell^{\prime}}}\right) \geq \mathcal{E}(u, u),
$$

as required in the generalized Mosco-convergence. In fact, by applying

$$
\mathcal{E}^{(\delta)}\left(\frac{1}{n} \sum_{\ell^{\prime}=1}^{n}\left(u_{k_{\ell^{\prime}}}\right)_{\ell^{\prime}}, \frac{1}{n} \sum_{\ell^{\prime}=1}^{n}\left(u_{k_{\ell^{\prime}}}\right)_{\ell^{\prime}}\right)^{1 / 2} \leq \frac{1}{n} \sum_{\ell^{\prime}=1}^{n} \mathcal{E}^{(\delta)}\left(\left(u_{\ell_{\ell^{\prime}}}\right)_{\ell^{\prime}},\left(u_{k_{\ell^{\prime}}}\right)_{\ell^{\prime}}\right)^{1 / 2},
$$

we can derive

$$
\begin{aligned}
\liminf _{k \rightarrow \infty} \mathcal{E}_{k}\left(u_{k}, u_{k}\right) & \geq \lim _{\ell^{\prime} \rightarrow \infty} \mathcal{E}_{k_{\ell^{\prime}}}^{(\delta)}\left(u_{k_{\ell^{\prime}}}, u_{k_{\ell^{\prime}}}\right) \\
& \geq(1-\delta)^{3} \limsup _{\ell^{\prime} \rightarrow \infty} \mathcal{E}^{(\delta)}\left(\left(u_{k_{\ell^{\prime}}}\right)_{\ell^{\prime}},\left(u_{k_{\ell^{\prime}}}\right)_{\ell^{\prime}}\right) \\
& \geq(1-\delta)^{3} \lim _{n \rightarrow \infty} \mathcal{E}^{(\delta)}\left(\frac{1}{n} \sum_{\ell^{\prime}=1}^{n}\left(u_{k_{\ell^{\prime}}}\right)_{\ell^{\prime}}, \frac{1}{n} \sum_{\ell^{\prime}=1}^{n}\left(u_{k_{\ell^{\prime}}}\right)_{\ell^{\prime}}\right) \\
& \geq(1-\delta)^{3} \mathcal{E}^{(\delta)}(u, u)
\end{aligned}
$$

from (3).

Since these inequalities hold valid for any $\delta>0$, the required inequality for the condition (a) has been validated.

The rest of the section is devoted to verify the other condition for the generalized Moscoconvergence. Namely, we will show that, for any $u \in L^{2}\left(F ; \mu_{0}\right)$, there exists a KS-strongly convergent sequence $\left\{u_{k}\right\}$ with the KS-strong limit $u$ satisfying $\lim \sup _{k \rightarrow \infty} \mathcal{E}_{k}\left(u_{k}, u_{k}\right) \leq$ $\mathcal{E}(u, u)$ as in (iv)-(b) in Definition 2.1 in [6].

We first note that we may assume $u$ in $\mathcal{F}$. By the definition of the smallest closed extension $\mathcal{E}$ of $\mathcal{E}_{0}$, for any $u \in \mathcal{F}$, we can take the sequence $\left\{\varphi_{n}\right\}$ in the family of locally constant function on $\boldsymbol{Z}_{p}$,

$$
\lim _{n \rightarrow \infty} \mathcal{E}\left(\varphi_{n}, \varphi_{n}\right)=\mathcal{E}(u, u) \quad \text { and } \quad \lim _{n \rightarrow \infty}\left\|\left.\varphi_{n}\right|_{F}-u\right\|_{L^{2}\left(F ; \mu_{0}\right)}=0 .
$$

By Proposition 1.1, Corollary 1.2 and the vague convergence of $\left\{\mu_{k}\right\}$ to $\mu_{0}$, there exists some $k_{1}>0$ such that for $k>k_{1}$

$$
\left|\left\|\varphi_{1}-\varphi_{0}\right\|_{L^{2}\left(\boldsymbol{Z}_{p} ; \mu_{k}\right)}-\left\|\varphi_{1}-\varphi_{0}\right\|_{L^{2}\left(F ; \mu_{0}\right)}\right|<\frac{1}{2}
$$

and

$$
\left|\mathcal{E}_{k}\left(\varphi_{1}, \varphi_{1}\right)-\mathcal{E}\left(\varphi_{1}, \varphi_{1}\right)\right|<\frac{1}{2} .
$$

Here and the sequel, the domain of the measure $\mu_{0}$ is viewed as the family of topological Borel sets in $\boldsymbol{Z}_{p}$. For some $k_{2}>0, k>k_{2}$ implies that

$$
\left|\left\|\varphi_{2}-\varphi_{0}\right\|_{L^{2}\left(Z_{p} ; \mu_{k}\right)}-\left\|\varphi_{2}-\varphi_{0}\right\|_{L^{2}\left(F ; \mu_{0}\right)}\right|<\frac{1}{2^{2}}
$$




$$
\left|\left\|\varphi_{2}-\varphi_{1}\right\|_{L^{2}\left(\boldsymbol{Z}_{p} ; \mu_{k}\right)}-\left\|\varphi_{2}-\varphi_{1}\right\|_{L^{2}\left(F ; \mu_{0}\right)}\right|<\frac{1}{2^{2}}
$$

and

$$
\left|\mathcal{E}_{k}\left(\varphi_{2}, \varphi_{2}\right)-\mathcal{E}\left(\varphi_{2}, \varphi_{2}\right)\right|<\frac{1}{2^{2}} .
$$

By repeating this procedure, for any positive integer $j$, there exists $k_{j}$ such that $k>k_{j}$ implies

$$
\left|\left\|\varphi_{j}-\varphi_{i}\right\|_{L^{2}\left(\boldsymbol{Z}_{p} ; \mu_{k}\right)}-\left\|\varphi_{j}-\varphi_{i}\right\|_{L^{2}\left(F ; \mu_{0}\right)}\right|<\frac{1}{2^{j}}
$$

for any positive integer $i$ with $i<j$ and

$$
\left|\mathcal{E}_{k}\left(\varphi_{j}, \varphi_{j}\right)-\mathcal{E}\left(\varphi_{j}, \varphi_{j}\right)\right|<\frac{1}{2^{j}}
$$

for any integer $k$ with $k>k_{j}$. For any $K$, there exists $i_{K}$ such that $i, j \geq i_{K}$ implies $\| \varphi_{j}-$ $\varphi_{i} \|_{L\left(\mathbf{Z}_{p} ; \mu_{0}\right)}<\frac{1}{2^{K}}$. Accordingly, one sees that $j \geq i_{K}$ implies

which shows that

$$
\left\|\varphi_{j}-\varphi_{i_{K}}\right\|_{L^{2}\left(F ; \mu_{0}\right)} \leq \frac{1}{2^{K}}
$$

$$
\left\|\varphi_{j}-\varphi_{i_{K}}\right\|_{L^{2}\left(Z_{p} ; \mu_{k}\right)} \leq\left\|\varphi_{j}-\varphi_{i_{K}}\right\|_{L^{2}\left(F ; \mu_{0}\right)}+\frac{1}{2^{j}} \leq \frac{1}{2^{K}}+\frac{1}{2^{j}},
$$

for any integers $j$ with $j>i_{K}$ and $k>k_{j}$.

We denote $\varphi_{i_{K}}$ by $\psi_{K}$ and define $\left\{u_{k}\right\}$ by $u_{k}=\varphi_{j}$ for $k_{j+1} \geq k>k_{j}$. Then for sufficiently large $k$,

which shows

$$
\left\|u_{k}-\psi_{K}\right\|_{L^{2}\left(Z_{p} ; \mu_{k}\right)} \leq \frac{1}{2^{j}}+\frac{1}{2^{K}}
$$

$$
\limsup _{k \rightarrow \infty}\left\|u_{k}-\psi_{K}\right\|_{L^{2}\left(\boldsymbol{Z}_{p} ; \mu_{k}\right)} \leq \frac{1}{2^{K}} .
$$

Since $\lim \sup _{K \rightarrow \infty}\left\|u-\psi_{K}\right\|_{L^{2}\left(F ; \mu_{0}\right)}=0$, the sequence $\left\{u_{k}\right\}$ converges KS-strongly to $u$. By combining this with $\lim \sup _{k \rightarrow \infty} \mathcal{E}_{k}\left(u_{k}, u_{k}\right)=\mathcal{E}(u, u)$ obtained by (4) and (5), the generalized Mosco-convergence of the Dirichlet forms is verified.

We shall shift our attention to the tightness of the Hunt processes associated with the Dirichlet spaces. We see that our Dirichlet form $\mathcal{E}_{k}$ is covered by the framework in [11] with Kolmogorov's equation. In fact, $\boldsymbol{Z}_{p}$ is described as the disjoint union of finitely many balls $\left\{K_{i}^{(m)}\right\}$ with radius $p^{-m}$ and the value $\mathcal{E}_{k}\left(1_{K_{i}^{(m)}}, 1_{K_{j}^{J(m)}}\right)$ of the symmetric bilinear form for distinct balls $K_{i}^{(m)}, K_{j}^{(m)}$ is given by $\mu_{k}\left(K_{i}^{(m)}\right) \int_{K_{j}^{(m)}} \rho_{k}(x, y) d \mu_{k}(y)$ determined independently by choice of $x \in K_{i}^{(m)}$. This shows that the Hunt process $\left\{X_{t}^{(k)}\right\}$ generated by the Dirichlet form $\mathcal{E}_{k}$ on $L^{2}\left(\boldsymbol{Z}_{p} ; \mu_{k}\right)$ admits a characterization by Kolmogorov's equations 


$$
\left\{\begin{array}{l}
\frac{d}{d t} P_{K_{f}^{(m)}, K_{i}^{(m)}}(t)=-\tilde{a}_{k}\left(K_{f}^{(m)}\right) P_{K_{f}^{(m)}, K_{i}^{(m)}}(t)+\sum_{j \neq f}^{\infty} \tilde{u}_{k}\left(K_{f}^{(m)}, K_{j}^{(m)}\right) P_{K_{j}^{(m)}, K_{i}^{(m)}}(t), \\
\frac{d}{d t} P_{K_{f}^{(m)}, K_{i}^{(m)}}(t)=-\tilde{a}_{k}\left(K_{i}^{(m)}\right) P_{K_{f}^{(m)}, K_{i}^{(m)}}(t)+\sum_{j \neq i}^{\infty} P_{K_{f}^{(m)}, K_{j}^{(m)}}(t) \tilde{u}_{k}\left(K_{j}^{(m)}, K_{i}^{(m)}\right),
\end{array}\right.
$$

with the coefficients $\tilde{u}_{k}\left(K_{f}^{(m)}, K_{j}^{(m)}\right)=\int_{K_{j}^{(m)}} \rho_{k}(x, y) d \mu_{k}(y)$ and $\tilde{a}_{k}\left(K_{f}^{(m)}\right)=\sum_{j \neq f}^{\infty}$ $\tilde{u}_{k}\left(K_{f}^{(m)}, K_{j}^{(m)}\right)=\int_{K_{f}^{(m)^{c}}} \rho_{k}(x, y) d \mu_{k}(y)$ determined independently by the choice of $x \in$ $K_{f}^{(m)}$.

This also shows that the exponential holding time of $\left\{X_{t}^{(k)}\right\}$ for the transition out of state $K_{f}^{(m)}$ is determined by the parameter $\tilde{a}_{k}\left(K_{f}^{(m)}\right)$. Namely, the exit time $\tau_{k, K_{f}^{(m)}}$ of $\left\{X_{t}^{(k)}\right\}$ from the ball $K_{f}^{(m)}$ is exponentially distributed with the parameter $\tilde{a}_{k}\left(K_{f}^{(m)}\right)$ under $P_{x}$ with $x \in K_{f}^{(m)}$. Accordingly, it admits the following estimate

$$
P_{x}\left(\tau_{k, K_{f}^{(m)}}<\delta\right)=1-\exp \left(-\tilde{a}_{k}\left(K_{f}^{(m)}\right) \delta\right) \leq \delta \int_{K_{f}^{(m)}{ }^{c}} \rho_{k}(x, y) d \mu_{k}(y)
$$

for any $x \in K_{f}^{(m)}$ and $\delta>0$ from which we can derive that

$$
\begin{aligned}
\sum_{K_{f}^{(m)} \subset \boldsymbol{Z}_{p}} \int_{K_{f}^{(m)}} P_{x}\left(\tau_{k, K_{f}^{(m)}}<\delta\right) d \mu_{k}(x) & \leq \delta \sum_{K_{f}^{(m)} \subset \boldsymbol{Z}_{p}} \int_{K_{f}^{(m)}} \int_{K_{f}^{(m) c}} \rho_{k}(x, y) d \mu_{k}(x) d \mu_{k}(y) \\
& =\frac{\delta}{2} \sum_{K_{f}^{(m)} \subset \boldsymbol{Z}_{p}} \mathcal{E}_{k}\left(1_{\left.K_{f}^{(m)}, 1_{K_{f}^{(m)}}\right) .}\right.
\end{aligned}
$$

Since the right-hand side does not exceed an arbitrarily given real number $\varepsilon>0$ for sufficiently small $\delta>0$ independently of the choice of sufficiently large $k$ as long as the radius $p^{-m}$ is fixed, the tightness of $\left\{X_{t}^{(k)}\right\}$ on any finite time interval follows from the general framework on Markov processes, as discussed in [6, Section 6].

For the main assertions in what follows, we replace the measure $\mu_{k}$ with $\mu_{k}(\cdot) / \mu_{k}\left(\boldsymbol{Z}_{p}\right)$ and denote the replaced measure again by $\mu_{k}$ for each $k=0,1,2, \ldots$ under the condition $\mu_{0}\left(\boldsymbol{Z}_{p}\right)>0$. In fact, all results obtained so far are valid after these replacements which can be performed with a positive probability in the cases based on the random fractal due to the results in [10]. When $\mu_{0}\left(\boldsymbol{Z}_{p}\right)=0$, we conventionally redefine $\mu_{0}$ as the trivial measure vanishing on $\boldsymbol{Z}_{p}$.

By applying the methods in the proofs of Theorem 2.1 and subsequent Corollary 2.1 in [6], we can obtain assertions similar to those in [6] on the resolvent $\left\{G_{\lambda}^{(k)}\right\}$ and the semi-group $\left\{P_{t}^{(k)}\right\}$ associated with the Dirichlet space $\left(\mathcal{E}_{k}, \mathcal{F}_{k}\right)$ for $k=1,2, \ldots$. Since the sequence of random Dirichlet forms has originally triggered our discussion, we can summarize the assertions in the following statements:

THEOREM 3.1. (i) The sequence $\left\{\mathcal{E}_{k}\right\}$ of Dirichlet forms is generalized Moscoconvergent to $\mathcal{E}$ as $k \rightarrow \infty$ with probability one under the condition $\mu_{0}\left(\boldsymbol{Z}_{p}\right)>0$, 
(ii) for any $\lambda>0$, the sequence $\left\{G_{\lambda}^{(k)}\right\}$ of the resolvent operators is $K S$-convergent to $G_{\lambda}$ as $k \rightarrow \infty$ with probability one under the condition $\mu_{0}\left(\boldsymbol{Z}_{p}\right)>0$,

(iii) for any $t>0$, the sequence $\left\{P_{t}^{(k)}\right\}$ of the semi-group operators is KS-convergent to $P_{t}$ as $k \rightarrow \infty$ with probability one under the condition $\mu_{0}\left(\boldsymbol{Z}_{p}\right)>0$.

COROLlary 3.2. The finite dimensional distribution of $\left\{X_{t}^{(k)}\right\}$ with initial distribution $\mu_{k}$ generated by the Dirichlet space $\left(\mathcal{E}_{k}, \mathcal{F}_{k}\right)$ weakly converges to the one of the Hunt process $\left\{X_{t}^{(0)}\right\}$ with initial distribution $\mu_{0}$ generated by the Dirichlet space $(\mathcal{E}, \mathcal{F})$, i.e., for any positive real numbers $t_{1}, t_{2}, \ldots, t_{\ell}$ with $t_{1}<t_{2}<\cdots<t_{\ell}$ and any real-valued continuous function $u$ on $\boldsymbol{Z}_{p}^{\ell}$

$$
\lim _{k \rightarrow \infty} E^{\mu_{k}}\left[u\left(X^{(k)}\left(t_{1}\right), \ldots, X^{(k)}\left(t_{\ell}\right)\right)\right]=E^{\mu_{0}}\left[u\left(X^{(0)}\left(t_{1}\right), \ldots, X^{(0)}\left(t_{\ell}\right)\right)\right]
$$

with probability one under the condition $\mu_{0}\left(\boldsymbol{Z}_{p}\right)>0$.

Let us denote the space of $\boldsymbol{Z}_{p}$-valued right continuous function on $[0, t]$ with left limits by $\boldsymbol{D}_{Z_{p}}([0, t])$. Then we have the following assertion similarly to [6]:

THEOREM 3.3. The probability law of the Hunt process $\left\{X_{t}^{(k)}\right\}$ under $P^{\mu_{k}}$ weakly converges to the one of $\left\{X_{t}^{(0)}\right\}$ under $P^{\mu_{0}}$ in $\boldsymbol{D}_{Z_{p}}([0, t])$ as $k$ tends to $\infty$ with probability one under the condition $\mu_{0}\left(Z_{p}\right)>0$.

\section{REFERENCES}

[ 1 ] S. Albeverio And W. Karwowski, A random walk on $p$-adics - the generator and its spectrum, Stochastic Process. Appl. 53 (1994), 1-22.

[ 2 ] S. N. Eithier And T. G. Kurtz, Markov processes: Characterization and convergence, John Wiley \& Sons Ltd., Hoboken, New Jersey, 1986.

[ 3 ] K. J. Falconer, Random Fractals, Math. Proc. Camb. Phil. Soc. 100 (1986), 559-582.

[4] K. J. FALCONER, Fractal geometry. Mathematical foundations and applications, John Wiley \& Sons Ltd., Chichester, New York, 1990.

[5] M. Fukushima, Y. Oshima And M. Takeda, Dirichlet forms and symmetric Markov processes, de Gruyter Stud. Math. 19, Walter de Gruyter, Berlin and Hawthorne, 1994.

[ 6 ] M. HinZ, Approximation of jump processes on fractals, Osaka J. Math. 46 (2009), 141-171.

[ 7 ] A. Imai, H. Kaneko And H. Matsumoto, A Dirichlet space associated with consistent networks on the ring of $p$-adic integers, $p$-Adic Numbers Ultrametric Anal. Appl. 3 (2011), 309-325.

[ 8 ] H. Kaneko, A class of spatially inhomogeneous Dirichlet spaces on the $p$-adic number field, Stochastic Process. Appl. 88 (2000), 161-174.

[9] H. KaneKo, A Dirichlet space on ends of tree and Dirichlet forms with a nodewise orthogonal property, Potential Anal. 41 (2014), 245-268.

[10] H. KANEKo AND W. NishiwaKi, A random fractal in the ring of $p$-adic integers, $p$-Adic Numbers Ultrametric Anal. Appl. 3 (2011), 74-80.

[11] W. KARWOWSKI AND R. Vilela-MENDES, Hierarchical structures and asymmetric stochastic processes on p-adics and adèles, J. Math. Phys. 35 (1994), 4637-4650.

[12] J. Kigami, Analysis on fractals, Cambridge Tracts in Math. 143, Cambridge University Press, Cambridge, 2001. 
[13] J. Kigami, Transitions on a noncompact Cantor set and random walks on its defining tree, Ann. Inst. Henri Poincaré Probab. Stat. 49 (2013), 1090-1129.

[14] T. Kumagai and K. T. Sturm, Construction of diffusion processes on fractals, $d$-sets, and general metric measure spaces, J. Math. Kyoto Univ. 45 (2005), 307-327.

[15] K. KUWAE AND T. Shioya, Convergence of spectral structures: a functional analytic theory and its applications to spectral geometry, Comm. Anal. Geom. 11 (2003), 599-673.

[16] A. Robert, A course in p-adic analysis, Grad. Texts in Math. 198, Springer-Verlag, New York, 2000.

[17] K. SuZUKI, Convergences and projection Markov property of Markov processes on ultrametric spaces, preprint.

[18] V. S. Vladimirov, I. V. Volovich and E. I. Zelenov, $p$-adic analysis and mathematical physics, Ser. Soviet East European Math. 1, World Scientific Publishing Co., Inc., River Edge, NJ, 1994.

[19] K. YAsudA, Additive processes on local fields, J. Math. Sci. Univ. Tokyo 3 (1996), 629-654.

DEPARTMENT OF MATHEMATICS

TOKYO UNIVERSITY OF SCIENCE

SHINJUKU, TOKYO 162-8061

JAPAN

E-mail address: stochos@rs.kagu.tus.ac.jp
DEPARTMENT OF MATHEMATICS

TOKYO UNIVERSITY OF SCIENCE

SHINJUKU, TOKYO 162-8061

JAPAN 